\title{
Ozone therapy in familial hypokalemic myopathy
}

\author{
Roberto Merante, Monica La Dolcetta \\ Scientific Society of Oxygen Ozone Therapy, Gorle (BG), Italy
}

\begin{abstract}
We report the case of a 56-year-old male with a familial hypokalemic paralysis suffering from progressive myopathy of the lower limbs. While episodes of paralysis were well controlled with oral potassium supplementation, muscle weakness in the lower limbs gave little response to rehabilitative therapies.

The patient underwent a series of treatments of ozone therapy alternated with large autohemoinfusion (GAEI) and rectal insufflation twice a week; GAEI was then maintained once a month. The muscle strength of the various muscle segments was evaluated during physiotherapy. A significant improvement in musculoskeletal strength and therefore in the response to rehabilitation treatments has been observed.

Oxygen-ozone therapy appears to be effective in increasing muscle strength in patients with genetic alterations of the potassium and calcium channels, thus making physiotherapeutic rehabilitation more effective.
\end{abstract}

\section{Introduction}

This case covers a period from February 2016 to January 2017. The patient - P. L. D. - was born in 1960 . The diagnosis was as follows: myopathic muscle suffering diagnosed with hypokalemic periodic paralysis (hypoPP) (25/05/2010); calcium muscle channelopathy $(31 / 03 / 2010)$.

Correspondence: Roberto Merante, Scientific Society of Oxygen Ozone Therapy, via Roma 69, 24020 Gorle (BG), Italy.

E-mail: merantero@alice.it

Key words: Ozone therapy; Familial hypokalemic myopathy; Major autohemoinfusion.

Received for publication: 11 September 2017.

Accepted for publication: 20 September 2017.

(C) Copyright R. Merante and M. La Dolcetta, 2017

Licensee PAGEPress, Italy

Ozone Therapy 2017; 2:7084

doi:10.4081/ozone.2017.7084

This article is distributed under the terms of the Creative Commons Attribution Noncommercial License (by-nc 4.0) which permits any noncommercial use, distribution, and reproduction in any medium, provided the original author(s) and source are credited.

\section{Case Report}

\section{Treatment}

Mr. P. L. D. from February 2016 to the present date, has carried out the following.

Ozone therapy sessions: 12 sessions were held twice a week, alternating one GAEI (180 mL of blood with $40 \mathrm{mg} / \mathrm{mL} \mathrm{O}_{3} / \mathrm{O}_{2}$ ) and one rectal insufflation at $30 \mathrm{mg} / \mathrm{mL}$. He then continued with one GAEI per month to date.

Approximately 2-3 manual therapy treatments focusing mainly on lumbar and hip areas per month. A program of exercises aimed at motor control and strengthening of the anterior inferior iliac spine and abdominal wall performed autonomously with constancy twice a week from February to August, and daily from August until today.

A 16-day hospitalization, from $02 / 08 / 2016$ to $17 / 08 / 2016$, at the Nemo Clinic Centre in Arenzano, Italy, where he was able to carry out, under the supervision of a physician, physiatrist and physiotherapist, a targeted rehabilitation program, which he continued at home.

\section{Pre- and post-oxygen-ozone therapy muscle strength evaluation}

There are no joint deficits or impaired muscle lengths (Table 1).

\section{Pre- and post-oxygen-ozone therapy functional evaluation}

\section{Walking}

February 2016: stand-alone walking without any rather insecure aids with poor pitch quality: the legs are stiff, the knee is hyperextended during the resting phase, the initial contact (the impact of the heel on the ground) is less for dorsiflection of the ankle limited by tibialis anterior muscle deficit; on the whole, the pace cycle times are not respected: the resting time of the limb is too long compared to the time of the pace. Balance is precarious and is easily lost if stressed by unexpected eternal forces; insecurity increases by walking on disconnected or unknown terrain (influence of psychological factor 1). He frequently falls due to knee failure (as he reported).

January 2017: qualitatively improved gait quality: greater fluidity in the pace, rebalancing the timing of the support phase and the phase of limb oscillation and greater safety; maintaining the quality of the wheelbase has also increased speed. The balance remains precarious during the journey on uneven terrain. Mr. P. L. D. uses the aid of a racquet when walking on slippery terrain otherwise it does not use aids and claims to feel safer during walking. Like one year ago he is able to carry the dog out (average size that tends to pull with moderate strength) independently going on routes he knows; the difficulty in maintaining a good and safe 
quality walking is increased if he carries weights (packages with minimum/medium load). Over the months, the number of episodes of falls fell significantly.

\section{Sit-to-stand}

February 2016: when reaching the upright position, it presents inverted patterns, first knee extension and then hip and trunk extension; large support base, which increases in width as the height of the chair decreases; support and massive help of the upper limbs is always necessary, with the impossibility of getting up even from higher seats without help.

The higher the seat, the higher the difficulty in reaching the upright position. When sitting down, the upper limbs need to be supported in order to slow down and control the descent: little muscular hold of the lower limbs (quadriceps).

January 2017: Patterns are always inverted but with better rebalancing: compensation for the hyperextension of the knees is activated after a good but not complete extension of the hips and the trunk; the support base has decreased in width. The need for help from the upper limbs remains; less effort is required to reach the position upright from lower seats.

\section{Climbing and descending stairs}

February 2016: impossibility to climb and descend the stairs without handrail or support of an upper limb; good achievement of the step with both feet, but in the push phase for the ascent of the steps Mr. P. L. D. uses hyperextension of the knees to compensate for muscle weakness (quadriceps) and then proceeds by incorrectly activating the muscles of the trunk and the help of the arm; the descent is not controlled and is always helped by the support of the upper limb.

January 2017: with a single support of the upper limb there is no improvement; while with double support (double stable handrail) the ascent and descent of the stairs is performed in an almost correct manner (fair compensation or strategies such as hyperextension of the knee are not activated) and controlled.

\section{Conclusions}

HypoPP ${ }^{1,2}$ is characterized by episodes of muscle paralysis that can last from a few hours to $24-48$ hours and are associated with a reduction in blood potassium levels. The prevalence is estimated at about 1/100,000. Paralysis very often affects the four limbs, thus causing quadriplegia. The main triggering factors are carbohydrate rich meals and rest after an effort. The onset of the disease usually occurs in the second decade of life. HypoPP is transmitted as an autosomal dominant character to incomplete penetration, particularly in females. Occasional cases and de novo mutations have been reported. About $70 \%$ of cases are associated with mutations in the gene encoding for the calcium channel of muscle cells, CACNA1S, and $10 \%$ of cases are due to mutations in the sodium channel gene of muscle cells, SCN4A. The electromyogram, in combination with stress and stress tests, reveals the lack of excitation of the muscle membrane and helps guide molecular diagnosis. Differential diagnosis arises with periodic thyrotoxic paralysis (see this term), which is associated with abnormal levels of thyroid hormone. Potassium supplementation and/or treatment with acetazolamide contribute to a significant decrease in the number of episodes and to a lesser extent in the resulting motor deficit.

The patient was diagnosed with myopathy in 2010, while familial hypokalemia had been known for some time already and treated with potassium supplementation to avoid paralysis. As we can evaluate from the physiotherapeutic control data there has been a significant improvement in strength and muscle endurance. This improvement allowed the patient to perform an intensive physiotherapeutic treatment, which was hampered by easy muscle fatigue. The result obtained remains unchanged one year after the start of therapy, with a clear reduction in restrictions on daily activities.

\section{References}

1. Stapleton LJ. Hypokalaemia periodic paralysis. Scott Med J 2017 (in press).

2. Weber F, Jurkat-Rott K, Lehmann-Horn F. Hyperkalemic periodic paralysis. In: Pagon RA, Adam MP, Ardinger HH, et al. (eds.) GeneReviews. Seattle (WA): University of Washington, Seattle; 1993-2017.

Table 1. Pre- and post-oxygen-ozone therapy muscle strength evaluation.

\begin{tabular}{|c|c|c|}
\hline Muscular groups & February 2016 & January 2017 \\
\hline Hip flexors (ileopsoas) & Left 2; Right 2 & Left 3; Right 2+ \\
\hline Hip extensors (hamstring, gluteus maximus) & Left 2 & Left 2 \\
\hline Adductors (gluteus medius and minimus, tensor fasciae latae) & Left $3+$; Right $3+$ & Right 4 \\
\hline Adductors (longus, brevis, magnus, pectineus, gracilis) & Left $3+$ & Left $4 \bullet$ Right 4 - \\
\hline Intrarotating (gluteus medius and minimus, tensor fasciae latae) & Left 4-; 4- & Left 4; Right 4 \\
\hline Extrarotating (piriformis, internal and external obturator, quadratus femoris) & Right 4 & Left 4+; Right 4- \\
\hline Knee flexors (hamstring) & Left 3-; Right 3- & Left 3 \\
\hline Knee extensors (quadriceps ) & Left 3-; Right 3- & Left 3 \\
\hline Ankle dorsiflexors (anterior tibialis) & Left 3-; Right 3+ & Left $4+$ \\
\hline Ankle plantar flexors (gastrocnemius and soleus) & Left $3+$ & Left $4, \bullet$ Right 4 \\
\hline Foot evertor (peroneus longus and brevis) & Left 4; Dx 4 & Left $4+$ \\
\hline Foot invertor (tibialis posterior) & Left 4- & Left 4-; 4- \\
\hline Extensor digitorum & Left 4 & Left 4 \\
\hline Flexor digitorum & Left 4 & Left 4-; Right 4+ \\
\hline
\end{tabular}

